\title{
Matayba guianensis (Sapindaceae): frugivoria por aves em remanescente de cerrado do Centro-Oeste brasileiro
}

\author{
Arly Porto de Oliveira ${ }^{1}$, Caio Graco Machado $^{2}$ \& Maria Rosângela Sigrist ${ }^{{ }^{*}}$
}

${ }^{1}$ Programa de Pós-Graduação em Biologia Vegetal, Departamento de Biologia, Centro de Ciências Biológicas e da Saúde, Universidade Federal do Mato Grosso do Sul, Campo Grande, Mato Grosso do Sul, Brasil.

${ }^{2}$ Laboratório de Ornitologia, Departamento de Ciências Biológicas, Universidade Estadual de Feira de Santana, Feira de Santana, Bahia, Brasil.

\begin{abstract}
Resumo - Frutos são recursos alimentares importantes, tanto para aves primariamente frugívoras quanto para espécies insetívoras e generalistas. Neste estudo, acompanhamos a frutificação de Matayba guianensis e registramos o consumo e a potencial dispersão de suas sementes por aves em remanescente urbano de cerrado em Campo Grande, Mato Grosso do Sul, de outubro de 2007 a setembro de 2008 e janeiro de 2009. Observações focais foram realizadas entre $06 \mathrm{~h} 00-10 \mathrm{~h} 00$ e $16 \mathrm{~h} 00-18 \mathrm{~h} 00$ no período de frutificação, totalizando $54 \mathrm{~h}$. Matayba guianensis frutificou por dois meses, na estação úmida, com maior produção de frutos imaturos em janeiro e maduros em fevereiro de 2008. Doze espécies de Passeriformes das famílias Tyrannidae (6 espécies), Thraupidae (3), Turdidae (2) e Passeridae (1) visitaram os frutos e/ou consumiram as sementes de M. guianensis, com 184 visitas registradas. A maioria das aves é onívora (8 espécies) e insetívora (3), com uma espécie nectarívora (Cyanerpes cyaneus). Tyrannidae predominou quanto à freqüência de visitas (87\%), quantidade de sementes consumidas $(96,2 \%)$ e versatilidade na obtenção das sementes, com predomínio da coleta em voo. Pitangus sulphuratus foi a espécie mais frequente (37,5\%) e a que consumiu maior número de sementes (48,3\%), seguida por Tyrannus melancholicus e Empidonomus varius. Estes tiranídeos são os principais potenciais dispersores de M. guianensis e, como a maioria das espécies desta família, são aves sinantrópicas, comuns em áreas urbanas e com grande habilidade para identificar e explorar novos recursos alimentares. As demais espécies de aves consumiram o arilo e descartaram as sementes próximas ou sob a planta-mãe, limitando sua dispersão.
\end{abstract}

Palavras-chave adicionais: Brasil, dispersão de sementes, Mato Grosso do Sul, Pitangus sulphuratus, Tyrannidae.

\begin{abstract}
Matayba guianensis (Sapindaceae): frugivory by birds in a remnant of cerrado in Central-West Brazil) - Fruits are important food resources for frugivorous birds and also for insectivores and omnivores. In this paper, we describe the fruit phenology, seed frugivory and potential dispersers of Matayba guianensis by birds in an urban savanna area in Mato Grosso do Sul state, Brazil, from October 2007 to September 2008 and January 2009. Focal observations were conducted between 6h00-10h00 AM and 4h00-6h00 PM, totalling $54 \mathrm{~h}$. Twelve species of Passeriformes of the families Tyrannidae (6 species), Thraupidae (3), Turdidae (2) and Passeridae (1) visited and/or consumed fruits of M. guianensis, with 184 records. Most birds are omnivores ( 8 species) and insectivores (3), and one nectarivore (Cyanerpes cyaneus). Tyrannidae predominated with the highest frequency of visits (87\%), consumption rates $(96.2 \%)$ and method of seed capture, with seeds predominantly gathered in flight. Pitangus sulphuratus was the most frequent visitor (37.5\%), consuming the greatest number of seeds (48.3\%), followed by Tyrannus melancholicus and Empidonomus varius. These Tyrannidae are the main potential seed dispersers of $M$. guianensis and as most Tyrannidae they are synanthropic species, common in urban habitats, having also great ability to identify and exploit new food resources. The other bird species consumed only the aril, discarding the seeds on or near the parent plant, thus limiting seed dispersal.
\end{abstract}

Additional key words: Brazil, Mato Grosso do Sul, Pitangus sulphuratus, seed dispersal, Tyrannidae.

Animais que consomem frutos e/ou sementes recebem retorno nutricional em troca da disseminação dos diásporos. Dessa maneira, a frugivoria pode estar associada à dispersão de sementes. Estima-se que um terço das espécies de aves em florestas tropicais utilize frutos como alimento (Snow 1981; Howe \& Smallwood 1982) e diversos estudos (e.g., Francisco \& Galetti 2001, 2002; Cazetta et al. 2002) têm registrado a utilização de uma espécie de planta por várias espécies de aves. Abrangendo grande amplitude taxonômica, diversidade trófica e morfológica, as aves podem contribuir de modo diferenciado para a

\footnotetext{
*Autor para correspondência: sigristster@gmail.com

Editor responsável: Clemens Schlindwein

Submetido: 8 abr. 2013; aceito: 25 out. 2013

Publicação inicial: 30 dez. 2013; versão final: 2 maio 2014
}

dispersão das sementes (Francisco \& Galetti 2001, 2002).

No Brasil, estudos sobre frugivoria por aves têm sido realizados sobretudo na Mata Atlântica da Região Sudeste (e.g., Gondim 2001; Cazetta et al. 2002; Guerra \& Marini 2002; Melo et al. 2003; Gridi-Papp et al. 2004; Almeida et al. 2006; Cazetta \& Galetti 2007; Pascotto 2007). Estudos em outras Regiões são escassos; podemos citar, por exemplo, trabalhos em campos rupestres da Bahia (Faustino \& Machado 2006; Bastos \& Machado 2007) e em florestas decíduas do Rio Grande do Sul (Krügel et al. 2006) e do Mato Grosso do Sul (Ragusa-Netto 2002). Em áreas de cerrado, os estudos também predominam no Sudeste, principalmente no estado de São Paulo (e.g., Francisco \& Galetti 2001, 2002; Marcondes-Machado 2002; Melo et al. 2003; Francisco et al. 2007). No Mato 
Grosso do Sul (MS), exceto quanto a Ficus calyptroceras (Miq.) Miq. (Moraceae) (Ragusa-Netto 2002), dados sobre frugivoria por aves são reportados em estudos sobre dieta e ecologia alimentar de psitacídeos e ranfastídeos (e.g., Ragusa-Netto \& Fecchio 2006) em mata de galeria e mata seca no Pantanal sul-matogrossense, que registram consumo de frutos de nove a 13 espécies de plantas.

Matayba Aubl. (Sapindaceae) é um gênero cosmopolita com aproximadamente 40 espécies que possuem sementes ariladas ornitocóricas (Barroso et al. 1999) e que, portanto, representam um recurso importante para a manutenção da avifauna. Espécies como M. elaeaginoides Radlk e M. guianensis Aubl. são indicadas na restauração ambiental por atrair frugívoros (Jordano et al. 2006). Entretanto, não existem estudos publicados enfocando o potencial de atração de frugívoros por espécies desse gênero e os dados disponíveis estão dispersos em estudos que abordam a dieta de aves. Em floresta atlântica do sudeste brasileiro, os frutos de $M$. elaeognoides são consumidos pelos contigídeos Capornis melanocephalus Wied, 1820 e Procnias nudicollis Vieillot, 1817 (Pizo et al. 2002). Além disso, Manhães (2003) relata o consumo do arilo das sementes de uma espécie de Matayba por três espécies de traupídeos, que não removem os diásporos das plantas.

Considerando a escassez de estudos publicados sobre frugivoria em espécies de Matayba, neste estudo, nós investigamos a frutificação e realizamos o levantamento da avifauna visitante e consumidora de sementes de Matayba guianensis Aubl. em remanescente de cerrado, registrando o comportamento de coleta e ingestão das sementes, número de sementes consumidas, frequência de visitação e tempo de permanência nas plantas.

\section{MATERIAL E MÉTODOS}

Área de estudo. Este estudo foi realizado em remanescente urbano de cerrado que faz parte da Reserva Particular do Patrimônio Natural da Universidade Federal de Mato Grosso do Sul $\left(20^{\circ} 27^{\prime} \mathrm{S}\right.$, $54^{\circ} 37^{\prime} \mathrm{W}$ ), em Campo Grande, Mato Grosso do Sul, de outubro de 2007 a setembro de 2008, com observações adicionais em janeiro de 2009. O fragmento possui cerca de 40 ha com vegetação composta principalmente por cerradão (sensu Oliveira-Filho \& Ratter 2002). O clima da região é do tipo Tropical Chuvoso de Savana - subtipo Aw (cf. Köppen 1948), com duas estações bem definidas, uma seca, de maio a setembro, e outra chuvosa, de outubro a abril.

Espécie estudada. Matayba guianensis é uma espécie arbórea (6-10 m alt.), monóica e pioneira, que ocorre em matas de galeria, matas mesófilas e cerradões (Ressel et al. 2004). Produz frutos do tipo cápsula loculicida, trilocular, com uma semente por lóculo. No fruto imaturo, o epicarpo é verde; no maduro, é avermelhado. As sementes são obovadas e possuem testa preta, envolvida na base por um arilo carnoso branco. Neste estudo, sementes provenientes de frutos maduros $(\mathrm{n}=15)$ de $M$. guianensis foram medidas com auxílio de paquímetro digital e possuem $8,7( \pm 1,0) \mathrm{mm}$ de comprimento e $6,4( \pm 1,0) \mathrm{mm}$ de largura. O material-testemunho de $M$. guianensis está depositado no Herbário CGMS da Universidade Federal de Mato Grosso do Sul, Campo Grande (CGMS 22386).

Fenologia de frutificação. $O$ período de frutificação de $M$. guianensis foi investigado mensalmente, de outubro de 2007 a setembro de 2008, em 10 indivíduos adultos marcados e escolhidos aleatoriamente na área de estudo. A fenofase de frutificação foi subdividida em fruto imaturo (ovário $\geq$ $2 \mathrm{~mm}$ compr. até adquirir o tamanho característico da espécie, com coloração verde, deiscente ou não) e fruto maduro (deiscente e com pericarpo avermelhado). A quantificação da frutificação foi realizada a partir do método proposto por Fournier (1974).

Avifauna. Os registros sobre as aves que visitaram e/ou consumiram os frutos de M. guianensis foram realizados com base em três plantas monitoradas, todas com grande quantidade de frutos (entre 700 e 1000 frutos). Cada planta foi monitorada por $12 \mathrm{~h}$, em sessões de observação indivíduo-focal entre 06h00$10 \mathrm{~h} 00$ e $16 \mathrm{~h} 00-18 \mathrm{~h} 00$, em janeiro e fevereiro de 2008 , com $18 \mathrm{~h}$ adicionais de monitoramento $(6 \mathrm{~h}$ em cada indivíduo) em janeiro de 2009, totalizando $54 \mathrm{~h}$. As observações foram feitas com auxílio de binóculo $7 \times$ $25 \mathrm{~mm}$, permanecendo o observador distante cerca de $15 \mathrm{~m}$ da planta observada.

Durante as observações foram registradas as espécies de aves que consumiram as sementes, o tempo e o padrão de visita (indivíduo solitário, aos pares ou em bandos), a quantidade de sementes consumidas por indivíduo, o tempo de permanência na planta e, sempre que possível, a distância de descarte das sementes. O número de sementes consumidas e o tempo de visita nas plantas foram determinados a partir de observações completas, onde as aves puderam ser observadas desde a chegada até a saída da planta.

As quatro táticas empregadas na captura dos diásporos foram agrupadas em duas categorias: em vôo (stalling) e pousada (hanging, picking e reaching) (modificada de Francisco \& Galetti 2001). A classificação taxonômica das aves e o status migratório seguiram as orientações do Conselho Brasileiro de Registros Ornitológicos (2011) e a determinação das guildas tróficas baseou-se em dados de literatura (Moojen et al. 1941; Hempel 1949; Schubart et al. 1965). 


\section{RESULTADOS}

Matayba guianensis frutificou por dois meses, na estação úmida, com maior produção de frutos imaturos em janeiro e maduros em fevereiro (figura 1). Os frutos geralmente ocorrem no ápice dos ramos, expostos fora da folhagem, em posição pendente a ligeiramente ereta.

Doze espécies de Passeriformes, pertencentes a quatro famílias, visitaram a planta e/ou consumiram as sementes de $M$. guianensis (Tabela 1), totalizando 184 visitas. A maioria das aves registradas é onívora (8 espécies.) e insetívora (3), com uma espécie nectarívora (Cyanerpes cyaneus). Indivíduos solitários $(61,5 \%)$ predominaram em relação aos pares $(23,1 \%)$ ou bandos $(15,4 \%)$ (Tabela 1$)$.

Indivíduos das espécies de Tyrannidae foram os mais freqüentes ( $87 \%$ das visitas) e os que consumiram maior quantidade de sementes ( $96,2 \%$ do consumo). Pitangus sulphuratus foi a espécie mais frequente (37,5\% dos registros) e a que consumiu maior número de sementes (48,3\% das sementes consumidas), seguida por Tyrannus melancholicus e Empidonomus varius (Tabela 1). Estes tiranídeos visitaram as plantas solitariamente, aos pares e em bandos (exceto Tyrannus albogularis, com padrão solitário de visita) (Tabela 1), sendo comuns os encontros agonísticos intraespecíficos. Estas espécies de aves visitaram esporadicamente frutos imaturos semiabertos, sendo capazes de remover o pericarpo com o bico e retirar a semente, que geralmente era ingerida no local (Tabela 2) ou podia ser carregada no bico (5 registros de Tyrannus melancholicus e 4 de Pitangus sulphuratus). Quanto a Tyrannus melancholicus, houve um registro de remoção da cápsula do fruto por um indivíduo adulto, deixando a semente a um filhote, que a removeu e em seguida a ingeriu. $O$ tempo de permanência nas plantas durante a visita variou de 15 seg. a $10 \mathrm{~min}$.

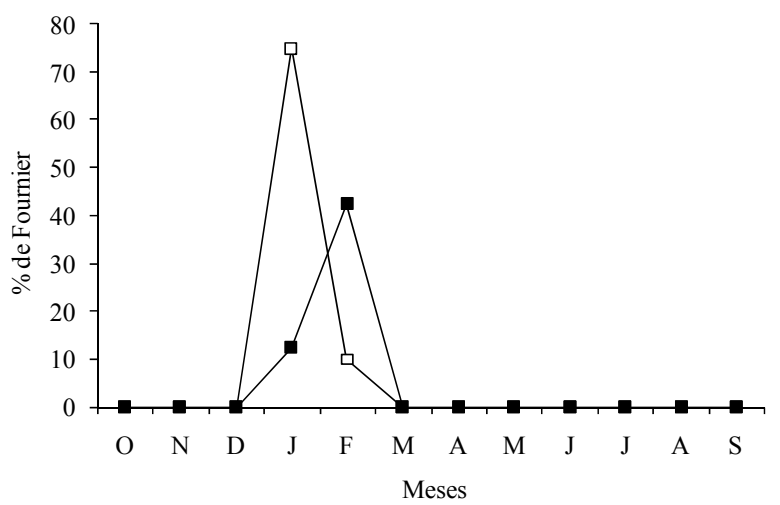

Figura 1. Ocorrência de frutos imaturos $(-\square-)$ e maduros $(-\mathbf{-}-)$ em 10 plantas de Matayba guianensis em remanescente de cerrado na Reserva Particular do Patrimônio Natural, Universidade Federal de Mato Grosso do Sul, Campo Grande, Mato Grosso do Sul, de outubro de 2007 a setembro de 2008.
Nas demais espécies, predominou o padrão de visita solitário (Tabela 1). Cyanerpes cyaneus e Dacnis cayana, após coletarem a semente, consumiram apenas $\mathrm{o}$ arilo, descartando a semente sob ou próxima à plantamãe (Tabela 2). Myiodynastes maculatus, Passer domesticus, Tangara sayaca, Turdus leucomelas e $T$. rufiventris não conseguiram ingerir as sementes coletadas, pois foram expulsos por indivíduos de Pitangus sulphuratus ou impedidos por interações agonísticas interespecíficas. Elaenia sp. apresentou um comportamento de coleta de sementes semelhante ao observado nas demais espécies de Tyrannidae.

Quatro táticas de forrageamento foram empregadas pelas espécies de aves, sendo que a maioria delas utilizou mais de uma tática (Tabela 2). As quatro espécies mais frequentes de Tyrannidae foram as mais versáteis na obtenção de frutos, com predominância de coleta dos frutos em voo (stalling) ou, quando pousadas, utilizaram o tipo picking, na qual a ave não assume nenhuma postura especial para acessar o fruto ou semente. Visita e coleta em voo também foi a principal técnica empregada pelos representantes de Turdidae, enquanto as espécies de Thraupidae realizaram visitas principalmente penduradas (reaching). Hanging foi a tática menos frequente entre as aves amostradas (Tabela 2).

\section{DisCuSSÃo}

Matayba guianensis frutifica e dispersa as sementes na estação úmida, período que, de maneira geral para as espécies de plantas em vegetação de cerrado, é mais adequado à germinação das sementes e estabelecimento das plântulas, que podem desenvolver sistema radicular ainda durante o período das chuvas (Oliveira 1998). Em fragmento urbano de floresta ombrófila densa e não sazonal, plantas de M. guianensis frutificaram por três meses, de novembro a janeiro (Santos et al. 2003), diferindo ligeiramente do registrado neste estudo. Variações na duração e no período de frutificação geralmente estão vinculados ao atraso ou antecipação da floração. $\mathrm{O}$ início e a duração das distintas fases de desenvolvimento de uma planta podem variar de ano para ano, dependendo das condições climáticas (Morellato et al. 2000). Além disso, fatores ambientais associados a mecanismos de regulação endógenos influenciam a frequência de floração, bem como o início da frutificação e amadurecimento das sementes (Bencke \& Morellato 2002).

Em Matayba guianensis os atributos de atração dos diásporos associados à dispersão por aves são a coloração avermelhada do pericarpo e o contraste do arilo branco com a testa escura, bem como a acessibilidade dos diásporos, pois os frutos ficam pendurados e expostos fora da folhagem. A espécie é generalista no tocante à estratégia de dispersão de sementes, pois as plantas produzem elevada quantidade de diásporos, atraindo ampla variedade de aves, muitas 
Tabela 1. Aves visitantes dos frutos de Matayba guianensis em remanescente de cerrado na Reserva Particular de Patrimônio Natural, Universidade Federal de Mato Grosso do Sul, Campo Grande, Mato Grosso do Sul, de janeiro e/ou fevereiro de 2008 e 2009 . Bm- Bando monoespecífico, FRU- frugívoro, INS- insetívoro, ONI- onívoro, P- par, S- solitário.

\begin{tabular}{|c|c|c|c|c|c|}
\hline \multirow[b]{2}{*}{ Espécie } & \multirow[b]{2}{*}{ Dieta } & \multicolumn{2}{|c|}{ Visita } & \multirow{2}{*}{$\begin{array}{l}\text { Número de } \\
\text { sementes } \\
\text { consumidas }\end{array}$} & \multirow{2}{*}{$\begin{array}{l}\text { Padrão } \\
\text { de visita }\end{array}$} \\
\hline & & Número & $\begin{array}{l}\text { Tempo } \\
\text { (seg.) }\end{array}$ & & \\
\hline \multicolumn{6}{|l|}{ PASSERIDAE } \\
\hline Passer domesticus Linnaeus, 1758 & ONI & 1 & 60,0 & 0 & $\mathrm{~S}$ \\
\hline \multicolumn{6}{|l|}{ TRHAUPIDAE } \\
\hline Cyanerpes cyaneus (Linnaeus, 1766) & NEC & 9 & 186,6 & 5 & $\mathrm{P} / \mathrm{S}$ \\
\hline Dacnis cayana Linnaeus, 1766 & ONI & 8 & 71,4 & 6 & $\mathrm{~S}$ \\
\hline Tangara sayaca Linnaeus, 1766 & ONI & 1 & 18,0 & 0 & $\mathrm{~S}$ \\
\hline \multicolumn{6}{|l|}{ TURDIDAE } \\
\hline Turdus leucomelas Vieillot, 1818 & ONI & 2 & 318,0 & 0 & $\mathrm{~S}$ \\
\hline Turdus rufiventris Vieillot, 1818 & ONI & 3 & 270 & 0 & $\mathrm{P} / \mathrm{S}$ \\
\hline \multicolumn{6}{|l|}{ TYRANNIDAE } \\
\hline Elaenia sp. & ONI & 2 & 249 & 1 & $\mathrm{~S}$ \\
\hline Empidonomus varius Vieillot, 1818 & INS & 40 & 188,4 & 47 & $\mathrm{P} / \mathrm{S}$ \\
\hline Myiodynastes maculatus Muller, 1776 & ONI & 2 & 78,0 & 0 & $\mathrm{~S}$ \\
\hline Pitangus sulphuratus Linnaeus, 1766 & ONI & 69 & 300,0 & 140 & $\mathrm{Bm} / \mathrm{P} / \mathrm{S}$ \\
\hline Tyrannus albogularis Burmeister, 1856 & INS & 9 & 83,4 & 14 & $\mathrm{~S}$ \\
\hline Tyrannus melancholicus Vieillot, 1819 & INS & 38 & 310,2 & 77 & $\mathrm{Bm} / \mathrm{P} / \mathrm{S}$ \\
\hline
\end{tabular}

Tabela 2. Táticas de forrageamento e comportamento de mandibulação de aves no consumo de frutos e/ou sementes de Matayba guianensis em remanescente de cerrado na Reserva Particular de Patrimônio Natural, Universidade Federal de Mato Grosso do Sul, Campo Grande, Mato Grosso do Sul, de janeiro e/ou fevereiro de 2008 e 2009. ES- engole a semente inteira; CA- come o arilo e descarta a semente sob ou próxima à planta-mãe; MD- mandíbula o diásporo sem remover o arilo e descarta a semente sob ou próximo à planta-mãe; n- número de registros.

\begin{tabular}{|c|c|c|c|c|c|c|c|}
\hline \multirow{3}{*}{ TÁXONS } & \multicolumn{4}{|c|}{ Táticas de captura $^{1}$} & \multirow{2}{*}{\multicolumn{3}{|c|}{$\begin{array}{l}\text { Comportamento } \\
\text { de mandibulação }\end{array}$}} \\
\hline & \multirow{2}{*}{$\begin{array}{c}\text { em voo } \\
\text { stalling }(\mathrm{n}) \\
\end{array}$} & \multicolumn{3}{|c|}{ pousado } & & & \\
\hline & & hanging (n) & picking $(\mathrm{n})$ & Reaching (n) & $\mathrm{ES}$ & $\mathrm{CA}$ & $\mathrm{MD}$ \\
\hline \multicolumn{8}{|l|}{ PASSERIDAE } \\
\hline Passer domesticus & - & - & 1 & - & - & - & - \\
\hline \multicolumn{8}{|l|}{ THRAUPIDAE } \\
\hline Cyanerpes cyaneus & - & - & - & 4 & - & $\mathrm{X}$ & - \\
\hline Dacnis cayana & 1 & 2 & - & 2 & - & $\mathrm{X}$ & - \\
\hline Tangara sayaca & - & - & - & 1 & - & - & $\mathrm{X}$ \\
\hline \multicolumn{8}{|l|}{ TURDIDAE } \\
\hline Turdus leucomelas & 1 & - & - & - & - & - & $X$ \\
\hline Turdus rufiventris & 2 & 1 & - & - & - & - & $\mathrm{X}$ \\
\hline \multicolumn{8}{|l|}{ TYRANNIDAE } \\
\hline Elaenia sp. & - & - & - & 1 & - & $\mathrm{X}$ & - \\
\hline Empidonomus varius & 33 & - & 4 & 4 & $\mathrm{X}$ & - & - \\
\hline Myiodynastes maculatus & - & - & - & 1 & - & - & $\mathrm{X}$ \\
\hline Pitangus sulphuratus & 55 & 1 & 14 & 6 & $\mathrm{X}$ & - & - \\
\hline Tyrannus albogularis & 15 & 1 & 1 & - & $\mathrm{X}$ & - & - \\
\hline Tyrannus melancholicus & 24 & - & 3 & 4 & $\mathrm{X}$ & - & - \\
\hline
\end{tabular}

${ }^{1}$ hanging $=$ todo o corpo da ave fica sob o poleiro, com a região ventral voltada para cima; picking $=$ captura os diásporos sem estender o corpo ou assumir posições especiais, reaching = estende o corpo abaixo ou acima do poleiro; stalling = captura o fruto em voo sem parar em frente a ele. 
delas consumidoras ocasionais de frutos (Mckey 1975; Pizo 1997; Francisco \& Galetti 2002). Produção de sementes relativamente pequena e em grande quantidade, como ocorre em M. guianensis, é atributo comum de espécies de sucessão secundária, funcionando como estratégia na colonização de áreas abertas (Howe 1993). Espécies pioneiras geralmente utilizam dispersores mais generalistas, como frugívoros onívoros ou insetívoros menos especializados (Mckey 1975; Snow 1981), que são mais flexíveis quanto aos hábitos alimentares (Marcondes-Machado 2002). Esta flexibilidade funciona como efeito-tampão durante flutuações no suprimento alimentar, que podem restringir a ocorrência de frugívoros, nectarívoros e mesmo insetívoros mais especializados (Willis 1979).

O predomínio de espécies frugívoras generalistas tem sido registrado em diversos estudos sobre frugivoria por aves em áreas de cerrado (e.g., Francisco \& Galetti 2001, 2002; Marcondes-Machado 2002; Melo et al. 2003). A riqueza da avifauna frugívora registrada no presente estudo é equivalente à reportada em outros estudos de caso sobre dispersão em áreas de cerrado (e.g., Francisco \& Galetti 2002; Melo et al. 2003). Tyrannidae é a família mais rica entre os Passeriformes americanos e é comumente registrada em estudos de frugivoria e dispersão de sementes. Embora consideradas primariamente insetívoras (Sick 1997), diversas espécies de tiranídeos incluem frutos e sementes na dieta (Marini \& Cavalcanti 1998), como por exemplo, diásporos de Ocotea pulchella Mart. (Francisco \& Galetti 2002), Talauma ovata A.St.-Hil. (Cazetta et al. 2002) e Trichilia spp. (Gondim 2001), dentre outras. Em áreas fragmentadas e antropizadas, há uma tendência de aumento no número de espécies Passeriformes, principalmente Tyrannidae, com simultânea redução dos não Passeriformes (Willis 1979).

Os tiranídeos Pitangus sulphuratus, Tyrannus melancholicus e Empidonomus varius são os principais potenciais dispersores de $M$. guianensis na área de estudo. Estas espécies apresentaram elevado número de visitas e diásporos consumidos, bem como comportamento de coleta adequado, adotando visitas em voo, sempre ingerindo os diásporos. Estes comportamentos aumentam a possibilidade das sementes serem regurgitadas ou defecadas longe da planta-mãe, onde a mortalidade por competição e/ou predação seria maior (Howe et al. 1985). Embora algumas espécies de aves tenham deixado cair sementes próximas ou sob a matriz, essas sementes podem ser secundariamente dispersadas por outros vetores, como formigas (e.g., Atta spp., Formicidae; Francisco \& Galetti 2001), sugerindo que estudos futuros investiguem o papel de formigas na dispersão de sementes de M. guianensis.

Comportamentos agonísticos intra e interespecíficos podem prejudicar a dispersão de sementes (Francisco \& Galetti 2001), podendo a espécie agressora impedir ou restringir a aproximação de outras espécies potencialmente dispersoras (Pizo 1997). Em M. guianensis, Pitangus sulphuratus foi a espécie mais agressora, fato que pode ter contribuído para o elevado número de visitas registradas e diásporos consumidos por esta espécie. As interações agonísticas parecem ter influenciado de modo negativo a dispersão de $M$. guianensis, pois impediram o consumo de suas sementes por cinco espécies de aves (Myiodynastes maculatus, Passer domesticus, Tangara sayaca, Turdus leucomelas e Turdus rufiventris).

Com base nos resultados obtidos aqui, podemos concluir que Matayba guianensis é uma espécie generalista quanto às aves que consomem suas sementes, característica importante em ambientes perturbados ou sob forte influência antrópica. Os tiranídeos, sobretudo Pitangus sulphuratus, Tyrannus melancholicus e Empidonomus varius, parecem ter papel importante na dispersão de suas sementes e podem ser considerados os principais potenciais dispersores dessa espécie. Embora não sejam espécies primariamente frugívoras, essas três espécies de aves são comuns em áreas antropizadas, inclusive áreas urbanas, e são hábeis na identificação e exploração de novos recursos alimentares.

\section{AGRAdeCIMENTOS}

CAPES e UFMS, pelas bolsas concedidas ao primeiro autor; Programa de Pós-graduação em Biologia Vegetal/UFMS, pelo apoio logístico; Gustavo Graciolli, Erica Hasui e Iêda Maria Novaes Ilha, pela leitura crítica do manuscrito; Arnildo Pott, pela revisão do abstract; Esther C. Bertazzonni e Jaqueline C. Vilas Boas, pelo auxílio na coleta de dados.

\section{REFERÊNCIAS}

Almeida, E.M.; Costa, P.F.; Buckeridge, M.S. \& Alves, M.A.S. 2006. Potential bird dispersers of Psychotria in an area of Atlantic forest on Ilha Grande, RJ, southeastern Brazil: a biochemical analysis of the fruits. Brazilian Journal of Biology 66: $1-8$.

Barroso, G.M.; Morim, M.P.; Peixoto, A.L. \& Ichaso, C.L.F. 1999. Frutos e Sementes: morfologia aplicada à Sistemática de Dicotiledôneas. Editora Universidade Federal de Viçosa, Viçosa.

Bastos, S.S. \& Machado, C.G. 2007. Visitantes frugívoros das espécies endêmicas Micranthocereus purpureus (Guerke) F. Ritter e Stephanocereus luetzelburgii (Vaupel) N. Taylor \& Eggli (Cactaceae) da Chapada Diamantina, Bahia, Brasil. Sitientibus série Ciências Biológicas 7: 314-318.

Bencke, C.S.C. \& Morellato, L.P.C. 2002. Comparação de dois métodos de avaliação da fenologia de plantas, sua interpretação e representação. Revista Brasileira de Botânica 25: 269-275.

Cazetta, E.; Rubim, P.; Lunardi, V.O.; Francisco, M.R. \& Galetti, M. 2002. Frugivoria e dispersão de sementes de Talauma ovata (Magnoliaceae) no sudeste brasileiro. Ararajuba 10: 199-206. 
Cazetta, E. \& Galetti, M. 2007. Frugivoria e especificidade por hospedeiros na erva-de-passarinho Phoradendron rubrum (L.) Griseb. (Viscaceae). Revista Brasileira de Botânica 30: 345351.

Conselho Brasileiro De Registros Ornitológicos. 2011. Lista primária de aves do Brasil. Disponível em: http://www.cbro.org.br/cbro/listapri.htm; aceso em 14 out. 2012.

Faustino, T.C. \& Machado, C.G. 2006. Frugivoria por aves em uma área de campo rupestre na Chapada Diamantina, BA. Revista Brasileira de Ornitologia 14: 137-143.

Francisco, M.R. \& Galetti, M. 2001. Frugivoria e dispersão de sementes de Rapanea lancifolia (Myrsinaceae) por aves numa área de cerrado do Estado de São Paulo, Sudeste do Brasil Ararajuba 9: 3-19.

Francisco, M.R. \& Galetti, M. 2002. Aves como potenciais dispersoras de sementes de Ocotea pulchella (Lauraceae) numa área de vegetação de cerrado do sudeste brasileiro. Revista Brasileira de Botânica 25: 11-17.

Francisco, M.R.; Lunardi; V.O. \& Galetti, M. 2007. Bird attributes, plant characteristics, and seed dispersal of Pera glabrata (Schott, 1858) (Euphorbiaceae) in a disturbed cerrado area. Brazilian Journal of Biology 67: 627-634.

Fournier, L.A. 1974. Un método cuantitativo para la medición de características fenológicas en arbores. Turrialba 4: 22-423.

Gondim, M.J.C. 2001. Dispersão de sementes de Trichilia spp. (Meliaceae) por aves em um fragmento de mata mesófila semidecídua, Rio Claro, SP, Brasil. Ararajuba 9: 101-112.

Gridi-Papp, C.O.; Gridi-Papp, M. \& Silva, W.R. 2004 Differential fruit consumption of two Melastomataceae by birds in Serra da Mantiqueira, southeastern Brazil. Ararajuba 12: 7 13.

Guerra, J.G. \& Marini, M.A. 2002. Bird frugivory on Struthanthus concinnus (Loranthaceae) in Southeastern Brasil. Ararajuba 10: 187-192.

Hempel, A. 1949. Estudo da alimentação natural de aves silvestres do Brasil. Arquivos do Instituto Biológico 19: 237-268.

Howe, H.F. 1993. Specialized and generalized dispersal systems: where does the "paradigm" stand? Vegetatio 107/108: 3-13.

Howe, H.F. \& Smallwood, J. 1982. Ecology of seed dispersal. Annual Review of Ecology and Systematics 13: 201-228.

Howe, H.F.; Schupp, E. \& Westley, L.C. 1985. Early consequences of seed dispersal for a neotropical tree Virola surinamensis. Ecology 66: 781-791.

Jordano, P.; Galetti, M.; Pizo, M.A. \&. Silva, W.R. 2006. Ligando frugivoria e dispersão de sementes à Biologia da Conservação. In: C.F.D. Rocha, H.G. Bergallo, M.A.S. Alves \& M. van Sluys (eds.). Biologia da Conservação: essências. RiMa Editora, São Carlos, p.411-436.

Köppen, W. 1948. Climatologia. Fundo de Cultura Econômica, Buenos Aires.

Krügel, M.M.; Burger, M.I. \& Alves, M.A. 2006. Frugivoria por aves em Nectandra megapotamica (Lauraceae) em uma área de Floresta Estacional Decidual no Rio Grande do Sul, Brasil. Iheringia Série Zoológica 96: 17--24.

Mckey, D. 1975. The ecology of coevolved seed dispersal systems. In: L.E. Gilbert \& P.H. Raven (eds.), Coevolution of Animals and Plants. University of Texas Press, Austin, p. 159-191.
Manhães, M.A. 2003. Dieta de traupíneos (Passeriformes, Emberizidae) no Parque Estadual do Ibitipoca, Minas Gerais, Brasil. Iheringia série Zoológica 93: 59-73.

Marcondes-Machado, L.O. 2002. Comportamento alimentar de aves em Miconia rubiginosa (Melastomataceae) em fragmento de cerrado, São Paulo. Iheringia série Zoológica 92: 97-100.

Marini, M.A. \& Cavalcanti, R.B. 1998. Frugivory by Elaenia flycatchers. Hornero 15: 47-50.

Melo, C.; Bento, E.C. \& Oliveira, P.E. 2003. Frugivory and dispersal of Faramea cyanea (Rubiaceae) in cerrado woody plant formations. Brazilian Journal of Biology 63: 75-82.

Moojen, J.; Carvalho, J.C. \& Lopes, H.S. 1941. Observações sobre o conteúdo gástrico das aves brasileiras. Memórias do Instituto Oswaldo Cruz 36: 405-444.

Morellato, P.C.; Talora, D.C.; Takahashi, A.; Bencke, C.C.; Romera, E.C. \& Zipparro, V.B. 2000. Phenology of Atlantic rain forest trees: a comparative study. Biotropica. 32: 811-823.

Oliveira, P.E.A.M. 2008. Fenologia e biologia reprodutiva das espécies de Cerrado. In: S.M. Sano, S.P. Almeida \& J.F. Ribeiro (orgs), Cerrado: ecologia e flora. Embrapa, Brasília, p.275290.

Oliveira-Filho, A.T. \& Ratter, J.A. 2002. Vegetation physiognomies and woody flora of the Cerrado biome. In: P.S. Oliveira \& R.J. Marquis (eds.), The Cerrados of Brazil: ecology and natural history opf a neotropical savanna. Columbia University Press, Nova York, p 91-120.

Pascotto, M.C. 2006. Avifauna dispersora de Alchornea glandulosa (Euphorbiaceae) em uma área de mata ciliar no estado de São Paulo. Revista Brasileira de Ornitologia 14: 291-296.

Pascotto, M.C. 2007. Rapanea ferruginea (Ruiz \& Pav.) Mez. (Myrsinaceae) como uma importante fonte alimentar para as aves em uma mata de galeria no interior do Estado de São Paulo. Revista Brasileira de Zoologia 24: 735-741.

Pizo, M.A.; Silva, W.R.; Galetti, M. \& Laps, R. 2002. Frugivory in cotingas of the Atlantic Forest of southeast Brazil. Ararajuba 10: 177-185.

Pizo, M.A. 1997. Seed dispersal and predation in two populations of Cabralea canjerana (Meliaceae) in the Atlantic Forest of southeastern Brazil. Journal of Tropical Ecology 13: 559-578.

Ragusa-Netto, J. 2002. Fruiting phenology and consumption by birds in Ficus calyptroceras (Miq.) Miq. (Moraceae). Brazilian Journal of Biology 62: 339-346.

Ragusa-Netto, J. \& Fecchio, A. 2006. Plant food resources and the diet of a parrot community in a gallery forest of the Southern Pantanal (Brazil). Brazilian Journal of Biology 66: 1021-1032.

Ressel, K.; Guilherme, F.A.G.; Schiavini. I. \& Oliveira, P.E. 2004. Ecologia morfofuncional de plântulas de espécies arbóreas da Estação Ecológica do Panga, Uberlândia, Minas Gerais. Revista Brasileira de Botânica 27: 311-323.

Santos, R.; Leal-Filho, L.S. \& Citadini-Zanette, V. 2003. Reabilitação de ecossistemas degradados pela mineração de carvão a céu aberto em Santa Catarina, Brasil. Boletim Técnico da Escola Politécnica da USP 205: 1-20.

Scherer, A.; Maraschin-Silva, F. \& Baptista, L.R.M. 2007. Padrões de interações mutualísticas entre espécies arbóreas e aves frugívoras em uma comunidade de Restinga no Parque Estadual de Itapuã, RS, Brasil. Acta Botanica Brasilica 21: 203-212. 
Schubart, O.; Aguirre, A.C. \& Sick, H. 1965. Contribuição para o conhecimento da alimentação das aves brasileiras. Arquivos de Zoologia 12: 95-249.

Sick, H. 1997. Ornitologia Brasileira. Nova Fronteira, Rio de Janeiro.
Snow, D.W. 1981. Tropical frugivorous birds and their food plants: a world survey. Biotropica 13: 1-14.

Willis, E.O. 1979. The composition of avian communities in remanescent woodlots in southern Brazil. Papeis Avulsos Zoologia 33: 1-25. 